\title{
Method for offset-curve generation for sheet-metal design
}

\section{Ken Wentland and Debasish Dutta}

\begin{abstract}
Offset curves have many applications in computer-aided design and manufacturing. The paper deals with sheet-metal design, where section curves that lie on a design surface must be offset by one metal thickness. Standard techniques for offsetting curves and surfaces are usually too slow for interactive speeds. The paper presents a simple iterative method for computing offset points which can then be splined to generate the offset curve. The method is first developed for the determination of nondegenerate offset curves, and then modifications necessary for the handling of degenerate offset curves are discussed. Implemented examples for simple sheet-metal design using the proposed method are also presented.
\end{abstract}

Keywords: offset curves, sheet-metal design, CAD/CAM

In sheet-metal design, there are two sides of the metal, which are referred to as the inside of the metal and the outside of the metal. Only one side of the metal is designed, namely the inside of the metal, which may also be referred to as the design side or design surface. The designer typically constructs a surface model of the design surface, i.e. a collection of parametric patches which, when pieced together, represents the design surface. The surfaces are usually in parametric form (i.e. $x=f(u, v)$, $y=g(u, v), z=h(u, v))$. Although the outside of the metal lies one metal thickness from the inside of the metal, surfaces are not created to represent the outside of the metal. After creating the design surfaces, a designer cuts planar sections through the design surfaces. In

Design Laboratory, Department of Mechanical Engineering \& Applied Mechanics, University of Michigan, Ann Arbor, MI 48109, USA

$\mathrm{K}$ Wentland is currently with Body Engineering Division, Ford Motor Company, Dearborn, MI 48121, USA

Paper received: 3 September 1992. Revised: 3 January 1993 automobile-body design, all the sheet-metal parts are designed in body position with reference to a world coordinate system, and it is typical to cut planar sections every $100 \mathrm{~mm}$ for each axis on the world coordinate system. Additional sections may be cut for added clarity, and to show key areas on a drawing. At every section cut on the design surface, it is necessary to show the profile of that section which lies on the outside of the metal. Planar sections consisting of a profile showing the inside of the metal and a profile showing the outside of the metal are frequently used to display key areas, to design mating parts, and to show the clearance and interference conditions for mating parts.

A simple sheet-metal part (design surface) is shown in Figure 1 . It consists of three surface patches $S_{1}, S_{2}$ and $S_{3}$. The curves $C_{1}, C_{2}$ and $C_{3}$ represent the planar sections cut through the three surface patches. Currently, two methods, which we refer to as the offset-curve method and the offset-surface method, are used by designers.

In the offset-curve method, a designer offsets the curve $C_{1}$ in the cutting plane by an amount equal to the metal thickness (see Figure 2). This method gives erroneous results when the surface normal is not parallel to the cutting plane. If the surface normals are parallel to the cutting plane, the generated offset profile is correct, and it is exactly one metal thickness away from the design curve. For example, in Figure 2, the surface normals for $S_{1}$ are parallel to the cutting plane. Therefore, the true offset curve $P_{1}$ is $2 \mathrm{~mm}$ from $C_{1}$, as desired. However, the surfaces $S_{2}$ and $S_{3}$ do not have surface normals that are parallel to the cutting plane. Therefore, the true offset profile is more than $2 \mathrm{~mm}$ away from $C_{2}$ and $C_{3}$. The result of the offset-curve method is clearly incorrect for $C_{2}$ and $C_{3}$.

In the offset-surface method, the idea is first to offset the surfaces by the amount of the metal thickness, and then to intersect the offset surfaces with the cutting plane 


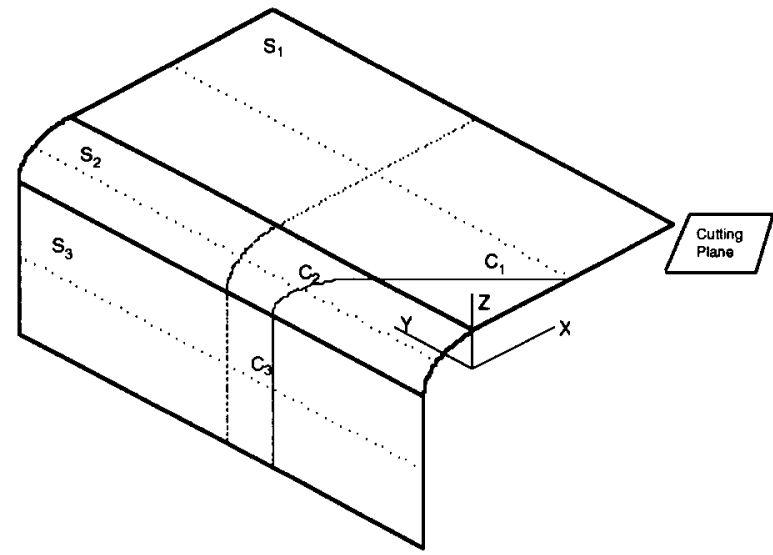

Figure 1 Simple sheet-metal design

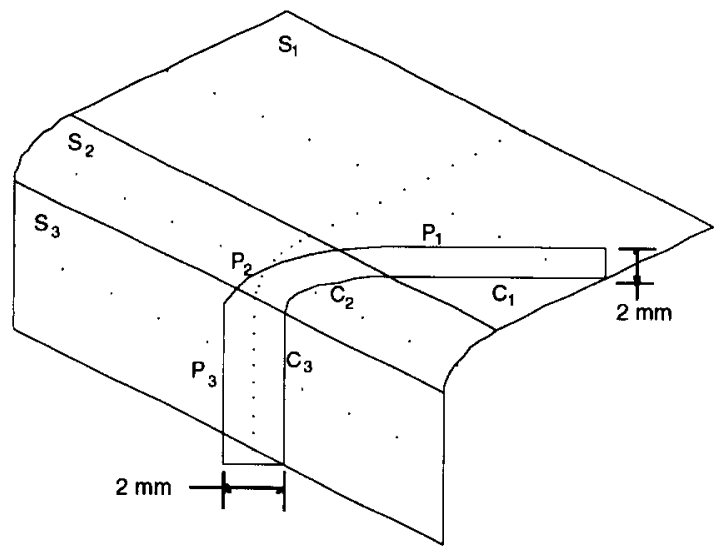

Figure 2 Planar offset for simple sheet-metal design (2 $\mathrm{mm}$ thickness)

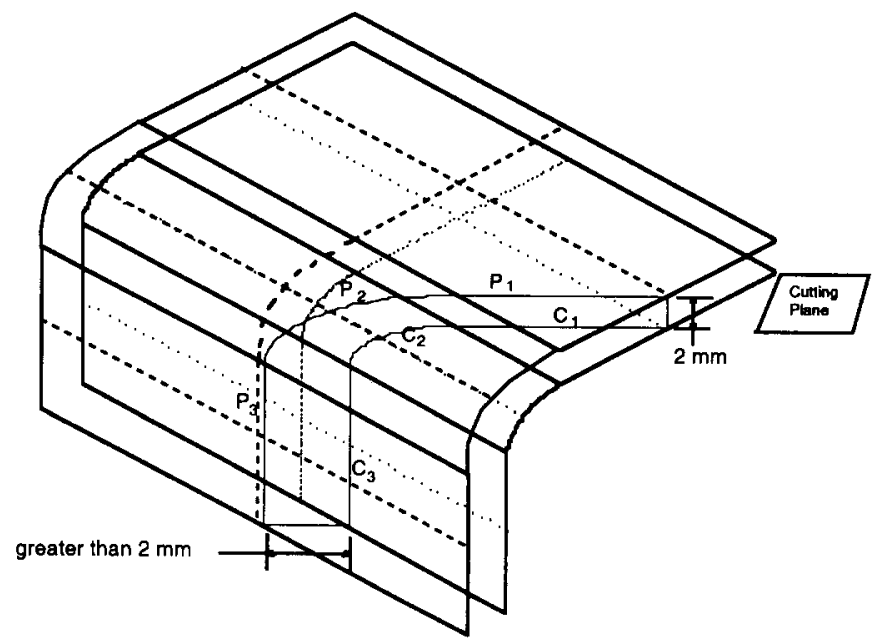

Figure 3 Profile offset by surface offset and intersection

to generate the offset profile. The offset surfaces can then be deleted. This method does give accurate results, and it is shown in Figure 3. However, the disadvantage of this method is that, for some surfaces, it is very difficult to obtain an accurate offset representation. Additionally, discontinuity in the design-surface boundaries can be magnified in the offset profile curve.

\section{PREVIOUS WORK}

The offsetting of curves and surfaces has received much attention in the field of computer-aided geometric design. Relevant literature dealing with methods for curve and surface offsetting is briefly discussed in this paper, and is not intended to comprise an exhaustive review. The difficulty in offsetting stems from the degree elevation that usually accompanies offsetting, and the handling of singularities $^{1}$. Tiller and Hanson studied various problems associated with the offsetting of planar curves, and presented some rules and procedures for computing their offsets ${ }^{2}$. Methods for the spline approximation of offset curves have been reported by Hoschek ${ }^{3}$. Farouki analyses the problem of computing offset surfaces, and presents methods for computing exact offsets of simple solids $^{4}$, and for approximating offsets of parametric surfaces by a composite of bicubic patches 5 . However, these methods are mathematically elegant but computationally intensive, and are therefore unsuitable for design applications that require interactive speeds. Further, these methods do not facilitate the modification of a degenerate surface to arrive at the closest approximation that is nondegenerate.

\section{DEGENERACIES IN DESIGN SURFACE}

In sheet-metal design, the problem often lies in avoiding degenerate cases, and, if they do occur, in how to deal with them. Figure 4 shows how a gap might occur while generating offsets to the section profile when two design surfaces meet with $\mathrm{C}^{0}$ (position) continuity. This is an exaggerated case of boundary discontinuity. The discontinuity in the design curve is magnified to cause a gap in the offset profile. There are three ways in which the offset profile can be remedied in such a situation. Figure 5 shows the three possible corrections. Note that only Figure $5 a$ is feasible for sheet-metal design, because, on Figure $5 b$ and $c$, the thickness of the sheet metal is not constant.

A cusp with a loop occurs when the design curve $C$ opens in the opposite direction. Figure 6 shows an example of a cusp with a loop (the curve normals are shown for better visualization). Typically, the offset curve $O$ would be trimmed to the point where the normals

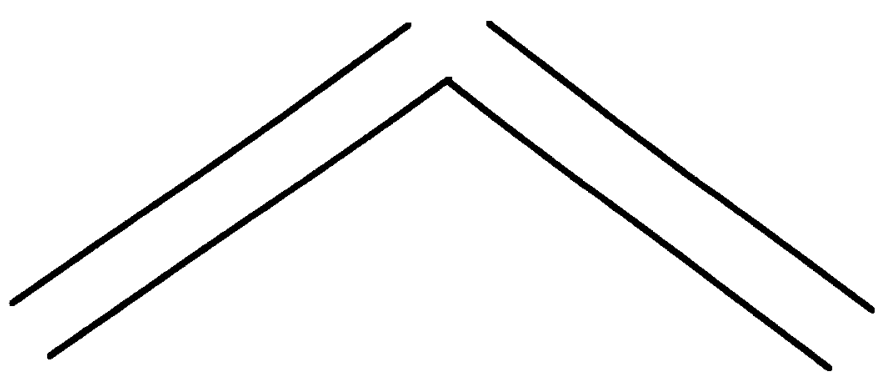

Figure 4 Boundary discontinuity 

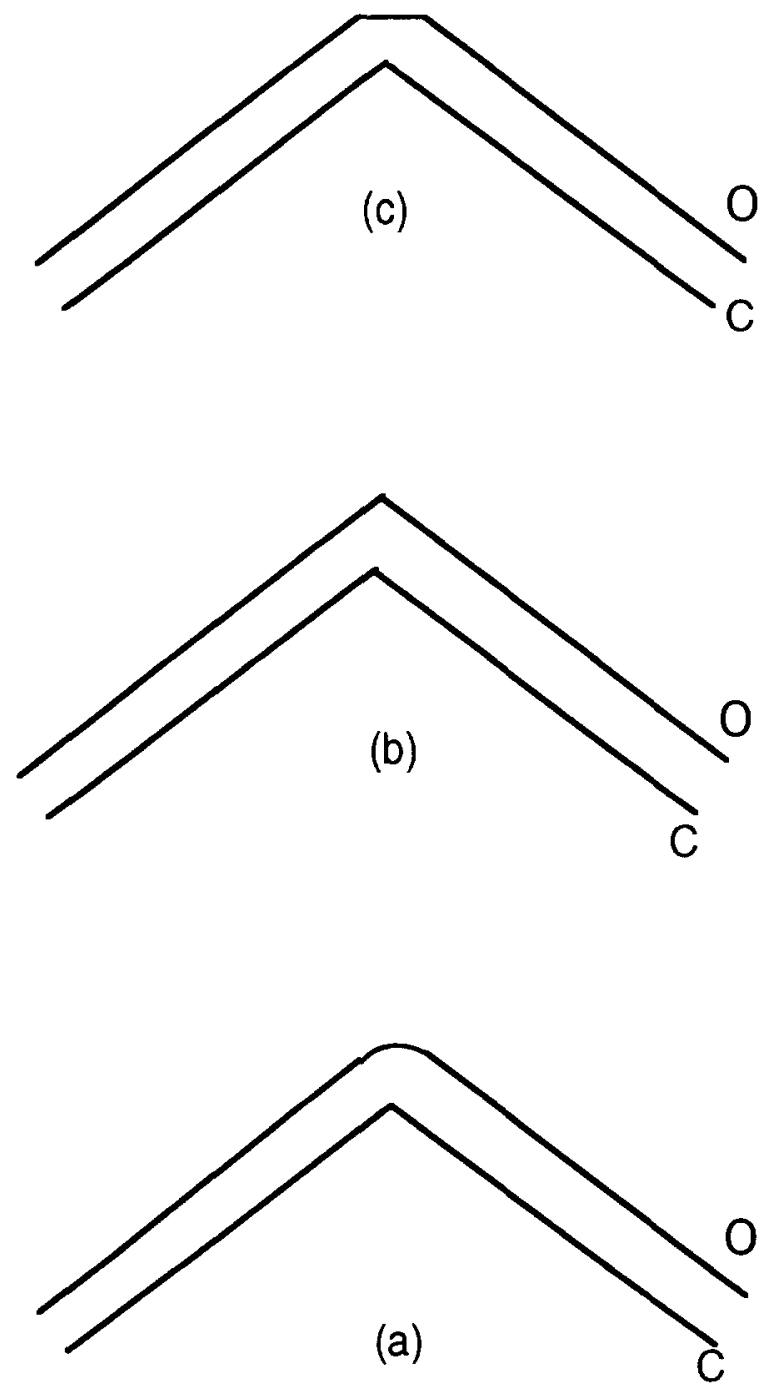

Figure 5 Possible approximations

intersect. For sheet-metal design, however, this is not sufficient, because the thickness is not constant.

The final degenerate case is that in which the thickness is greater than the radius of curvature. In this case, the normals cross, producing a cusp and loops. Figure 7 shows an example of this case. As with the previous case, constant thickness is lost when the offset curve $O$ is trimmed.

In summary, there are three degenerate cases: (a) $\mathrm{C}^{0}$ continuity on the design curve causing a gap in the offset curve, (b) $\mathrm{C}^{0}$ continuity on the design curve causing a cusp and loops in the offset curve, and (c) the thickness of the metal exceeds the radius of curvature, causing self-intersecting loops. Such degeneracies occur while offsetting curves as well as surfaces ${ }^{6}$.

\section{GENERATING POINTS ON OFFSET CURVE}

Consider the following problem. We are given a design surface $S$ (of a sheet-metal part) with a planar section, such as that shown in Figure 1 , cut by a plane $T$. We

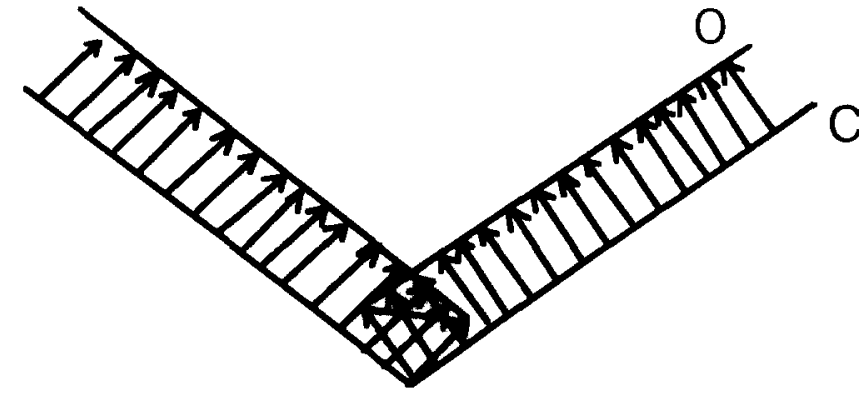

(a)

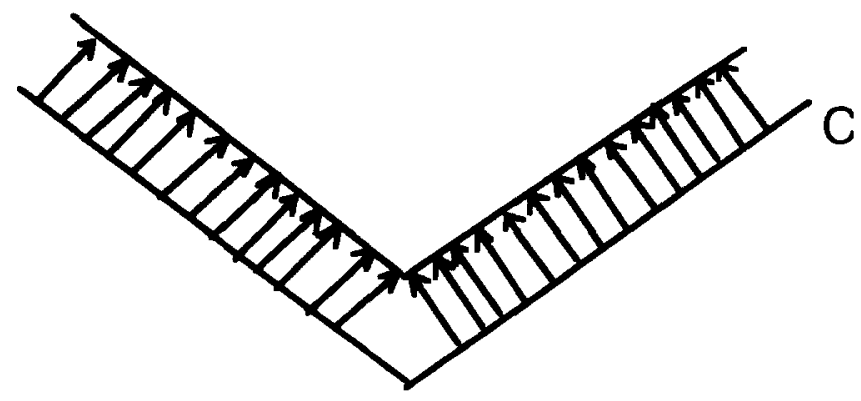

(b)

Figure 6 Cusp with loop; (a) offset curve with loop, (b) offset curve with loop trimmed

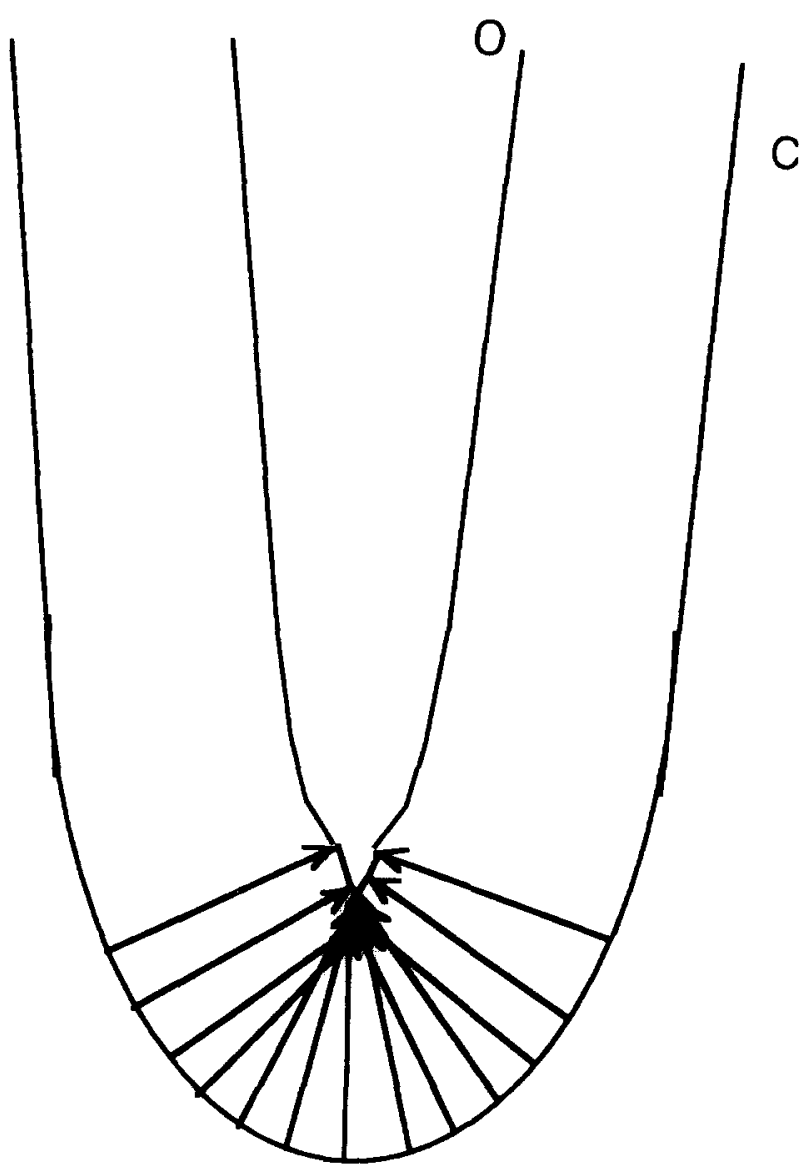

Figure 7 Metal thickness exceeds radius of curvature 
refer to the section curve as the design curve $C$. It is required to obtain the offset curve $P$ that corresponds to the design curve $C$ and meets the following criteria:

- Criterion 1: The offset curve $P$ lies in the cutting plane $T$ that contains the design curve $C$.

- Criterion 2: The offset curve $P$ is a constant thickness $t$ from the design surface $S$.

- Criterion 3: For every point $u$ on $C$, there is a corresponding point $v$ on $P$ which satisfies criteria 1 and 2 , and the line segment $(u, v)$ is normal to the curve $C$ in the cutting plane $T$. Therefore, if $C$ has $n$ data points, then so does $P$.

The offset-surface method described in the previous section (with Figure 3) generates the offset curve which satisfies the above criteria. However, because of computational problems associated with offsetting surfaces, it is rather difficult to implement for use in interactive design environments. A method is now proposed that satisfies the criteria $1-3$, but does not involve offsetting surfaces.

Intuitively, the existence of an offset curve $P$ satisfying the above criteria is guaranteed for every 'infinite' design surface. The constraint that the surface must be infinite is necessary to avoid a special case (see Figure 8). When the cutting plane $T$ intersects a design surface at a boundary that is not shared by another surface, it is possible that $P$ may not satisfy criterion 2 , and therefore also violate criterion 3 . Physically, this situation occurs at the 'edge' of the sheet-metal part. In this situation, the design surface has terminated, and it cannot be checked for thickness. However, this special case is of little practical consequence, since the area of the sheet metal is much larger than the thickness, and, more importantly, the design surfaces are typically overextended beyond the edge of the metal (i.e. the surface is bounded before it terminates).

Our approach to generating the offset curve $P(u)$ that satisfies the above criteria is to generate points on the offset curve. These points, when splined, give the offset profile. To generate offset points, we iteratively find the length of the surface normal on the design surface $S_{i}(r, s)$ such that the above criteria are satisfied. Let the offset curve be defined as follows:

$$
\mathbf{P}(u)=\mathbf{C}(u)+R(u) * \mathbf{N}_{1} \quad 0<u<1
$$

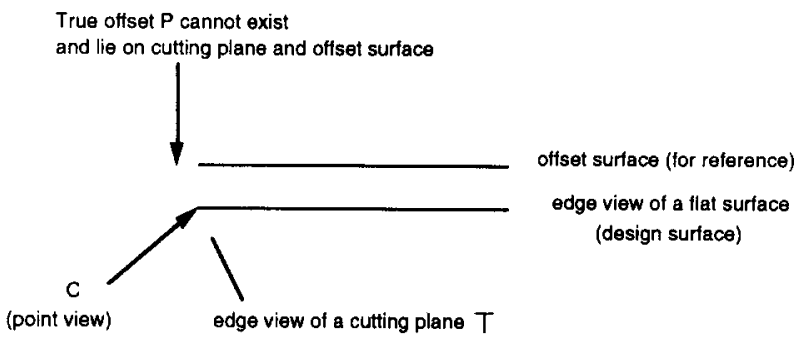

Figure 8 Special case

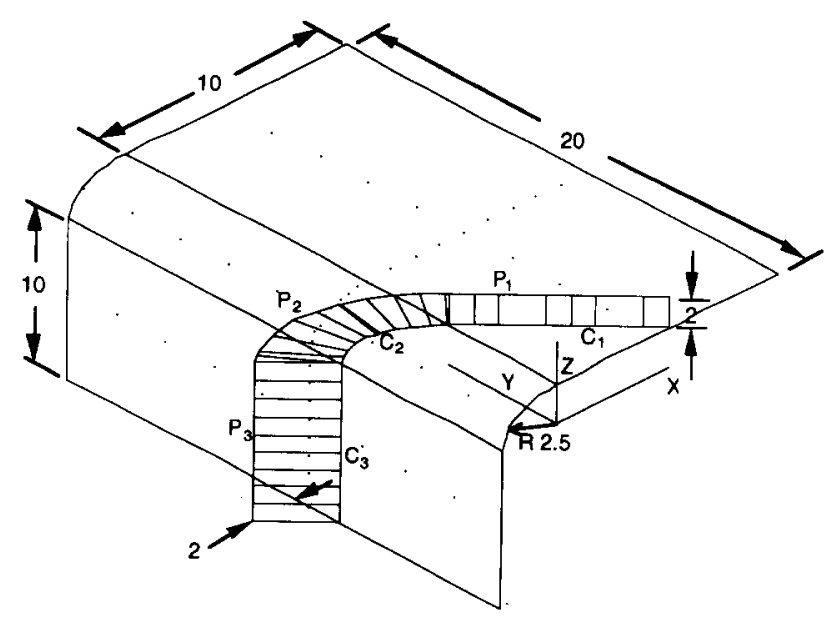

Figure 9 Method implementation with $R^{*} \mathbf{N}_{1}$

where $\mathbf{C}(\mathrm{u})$ is the design curve obtained by sectioning the design surface $S_{i}(r, s)$ with a cutting plane $T, R(u)$ is the offset distance in the cutting plane $T$, and $\mathbf{N}_{1}$ is the unit normal at $\mathbf{C}(u)$ in the cutting plane $T$. For each point on the design curve, the proposed method increases $R(u)$ iteratively until $\mathbf{P}(u)$ satisfies the offset criteria $1-3$. Mathematically, we have

$$
\mathbf{P}(u)=\mathbf{C}(u)+R(u) * \mathbf{N}_{1}
$$

and

$$
\mathbf{P}(u)=\mathbf{D}(u)+t * \mathbf{N}_{2}
$$

where $S_{i}(r, s)$ is the design surface corresponding to surface patches $i=1,2,3, \ldots, \mathbf{D}(u)$ is the footpoint of the perpendicular from $\mathbf{P}(u)$ to the design surface $S_{i}(r, s)$, $R(u)$ is the offset distance in cutting plane $T, t$ is the metal thickness, and $\mathbf{N}_{2}$ is the design-surface unit normal at $\mathbf{D}(u)$.

The important condition is that $\mathbf{D}(u)$ lies on the design surface, and we seek the minimum $R(u)$ that satisfies criteria $1-3$. Equations 1 and 2 must be solved iteratively. In general, we do this by setting the initial value of $R(u)=t$, and the subsequent values

$$
R(u)=R(u)+t-\|\mathbf{P}(u)-\mathbf{D}(u)\|
$$

until

$$
t-\|\mathbf{P}(u)-\mathbf{D}(u)\|<\varepsilon
$$

where $\varepsilon$ is the desired accuracy.

Figure 9 shows our implementation of this method, with $R * \mathbf{N}_{1}$ being shown for clarification. In Figure 10, the same section is shown, with $t * \mathbf{N}_{2}$. Thus, once Equations 1 and 2 are satisfied, $\mathbf{P}(u)$ can be represented by either equation. One advantage of using Equation 1 is that $R(u)$ can be varied from 0 to $R(u)$ to represent a 


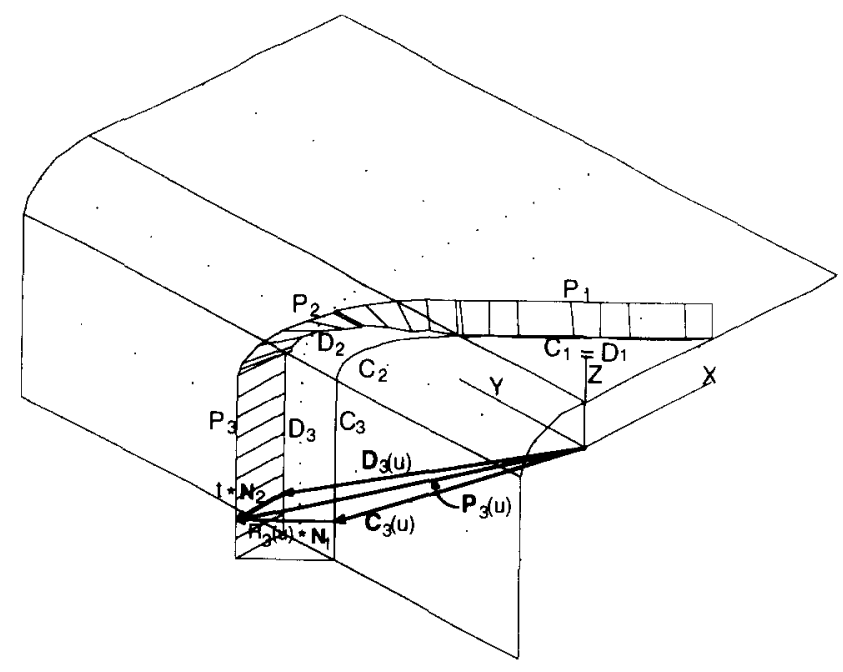

Figure 10 Method implementation with $t * \mathbf{N}_{2}$

planar face, or, if more sections are cut, a solid can also be represented.

Summarizing, an iterative procedure for generating points on the offset curve from a given design curve $\mathbf{C}(u)$ is as follows:

- Step l: Set the initial value of $R(u)=t$.

- Step 2: Compute $\mathbf{P}(u)$ using Equation 1.

- Step 3: Compute $\mathbf{D}(u)$, the footpoint of the perpendicular from $\mathbf{P}(u)$ to the design surface $S_{i}$. If $\mathbf{P}(u)-\mathbf{D}(u)<\varepsilon$, go to Step 5 .

- Step 4: Increment $R(u)$ using Equation 3. Go to Step 2.

- Step 5: Increment $u$. Get new point on the design curve $\mathbf{C}(u)$. Go to Step 1 .

For planar design surfaces, the procedure can be made more efficient, since the iterations are not necessary. Clearly, when the surface normal $\mathbf{N}_{2}$ at any point on the design curve $\mathbf{C}(u)$ is parallel to the cutting plane $T$, no further computations are required. When the design surface is planar, but the surface normal $\mathbf{N}_{2}$ is not parallel to the cutting plane $T$, the increment for $R(u)$ can be obtained directly from Equation 5 as follows (see also Figure (1).

$$
\left[\mathbf{P}^{\prime}-\mathbf{D}^{\prime}\right] / t=R(u) /[R(u)+x]
$$

where $\mathbf{P}^{\prime}$ and $\mathbf{D}^{\prime}$ are the offset-curve point and its footpoint on the design surface, respectively, corresponding to the initial value of $R(u)=t$. We solve for $x$, the required increment for $R(u)$. For nonplanar surfaces, however, the right-triangle analysis using initial and final footpoints is not possible, and we resort to the iteration.

\section{DISCUSSION}

The following aspects of the outlined method are considered in detail in this section:

- convergence issues,

- obtaining the offset profile from the offset points,

- the number of offset points that are necessary.

The overall method operates in a pointwise manner, as mentioned above. That is, for each point on the design curve, an offset-curve point is generated. The iteration is only necessary for the incrementation of $R(u)$, which is initially set to $t$ (one metal thickness). In Equation 4, $[\mathbf{P}(u)-\mathbf{D}(u)]$ is always positive, and, since the design surface is never ill behaved, always less than $t$.

Figure 12 shows the performance of this iterative method for the design surface shown in Figure 10. In particular, it compares the various $\varepsilon$ (i.e. the chosen levels of accuracy) with the number of iterations that were necessary for the 'worst point'. (The worst point refers to the point(s) that took the maximum number of iterations to reach the chosen $\varepsilon$ ).

In the method outlined above, we assume that the design curves (i.e. crosssections of the design surface) are provided to us as parametric curves $C_{i}(u), 0<u<1$. Note that the (planar intersection) computation of such design curves is straightforward, and not the subject of this paper. Hereafter, we deal explicitly with each point $\mathbf{C}\left(u^{*}\right)$ on the design curve, and, using the method outlined, obtain the corresponding point $\mathbf{P}\left(u^{*}\right)$ that lies on the offset curve. To obtain the offset-curve profile, the designer might choose to fit a curve through the offset points $\mathbf{P}(u)$ using known techniques (see, for example, Chapter 6 in Reference 7). In our implementation, for simplicity, we chose a piecewise-linear approximation of the offset profile.

Our method generates one point on the offset curve for each point selected from the design curve $\mathbf{C}(u)$. The total number of points chosen from the design curve depends on the designer's need for accuracy. Intuitively, for a chosen level of accuracy, the number of points necessary on the design curve increases with the algebraic degree of the curve. However, a designer is rarely

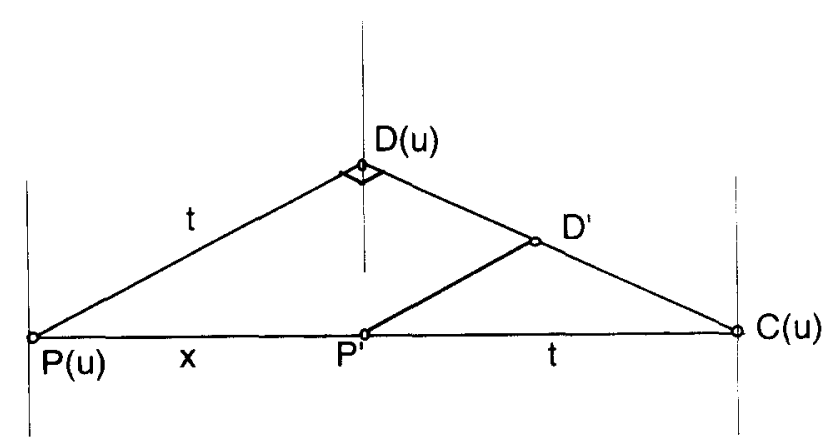

Figure 11 Increment for $R(u)$ obtained from Equation 5 


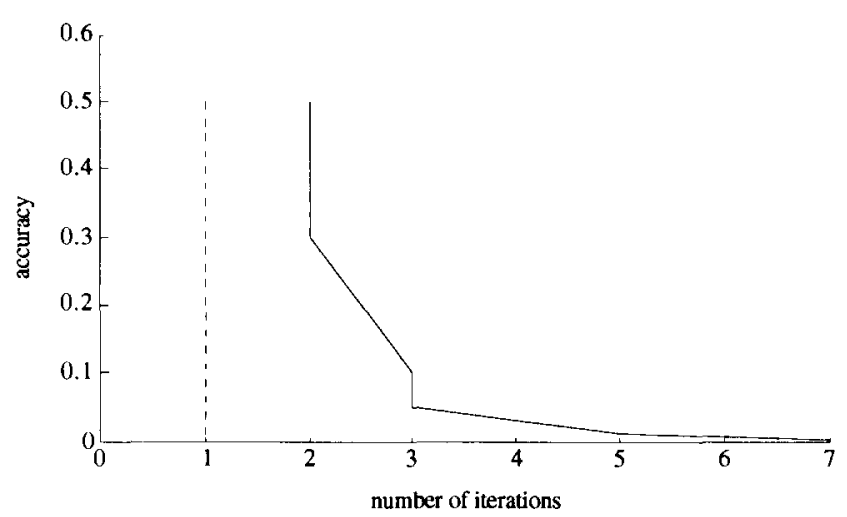

Figure 12 Performance of iterative method for design surface shown in Figure 10

concerned with, or aware of, the algebraic degrees of the parametric design surface. Generally, points are selected to be sufficiently close on the nonplanar design curves, while relatively few points suffice for planar sections. Consistently with current practice, the designer can select the number of points and the type of curve fit for the points to achieve a desired accuracy. In the example shown in Figure 18, we selected approximately 180 points (20 points per surface patch). Typically, the accuracy is checked by the designer visually comparing the offset profile to the profile of the design curve.

Finally, we note that there are no restrictions on the shape or continuity of the design surfaces for this method to work. However, the surface must be parameterizable, since we deal explicitly with points from the design curves. When there is only $\mathrm{C}^{0}$ continuity between surfaces, degeneracies arise, as shown in Figure 4. However, we do not deal with such degeneracies here, since, in sheet-metal design, the gaps are typically very small. Common practice is to connect the endpoints with a line, as shown in Figure $5 c$.

\section{HANDLING DEGENERATE CASES}

The degenerate cases shown in Figures 6 and 7 are addressed in this section. Here, the offset-curve normals cross, and self-intersecting loops occur in the offset curve. We describe a simple scheme to detect and rectify such situations. We consider the following criteria in the correction of the degenerate cases:

- Criterion 4: The offset-curve normals $\left[R(u) * \mathbf{N}_{1}\right]$ must not cross.

- Criterion 5: For each point on the design curve $\mathbf{C}(u)$, there must be a corresponding point on the offset curve $\mathbf{P}(u)$.

In Figure 13, the offset curve is on the opposite side of the sheet metal as compared with Figure 9, and the degeneracy is clear. In Figure 14, all the normals $\left[R(u) * \mathbf{N}_{1}\right]$ associated with the design curve $\mathbf{C}_{2}$ intersect, and therefore the entire offset curve $\mathbf{P}_{2}$ vanishes. Both of these cases occur where the value of $R(u)$ exceeds the radius of curvature.

The proposed method is to replace the offset-curve points $\mathbf{P}(u)$ at which the curve normals intersect (i.e. at the location at which the loop is trimmed off). In many situations, several of the points $\mathbf{P}(u)$ coincide. In Figure 14 , if $\mathbf{P}_{1}$ and $\mathbf{P}_{3}$ are trimmed to a point $\mathbf{P}_{2}, \mathbf{C}_{2}$ does not satisfy the constant-thickness criterion for an offset curve. The points of $\mathbf{C}_{2}$ are no longer a constant thickness $t$ away from its corresponding points on $\mathbf{P}_{2}$. The design curve $C_{2}$ must therefore be modified to satisfy this constraint. Physically, this means that the bend radius of the sheet metal connecting the two flat surfaces must be increased if the selected thickness $t$ is to be used. Alternatively, the selected thickness $t$ could be decreased to a value at which the design curve $\mathbf{C}_{2}$ does not need modification. In the following, we consider the first case only, since the second case is easy to handle by iterating on the thickness $t$ until the curve normals do not intersect.

A stepwise procedure to check for and correct the degeneracy described above is as follows:

- Step 1: Find the set of intersecting curve normals $\boldsymbol{\kappa}$.

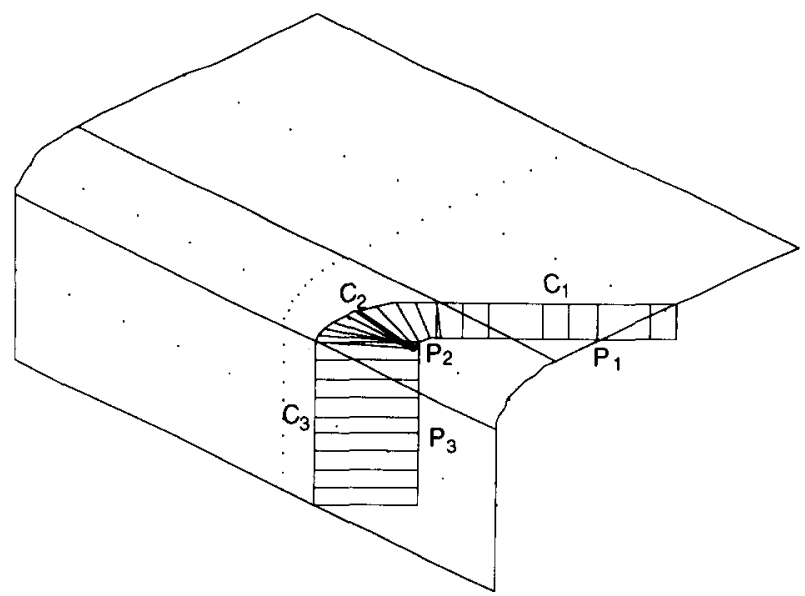

Figure 13 Degenerate case with offset curve on opposite side of meta in comparison with Figure 9

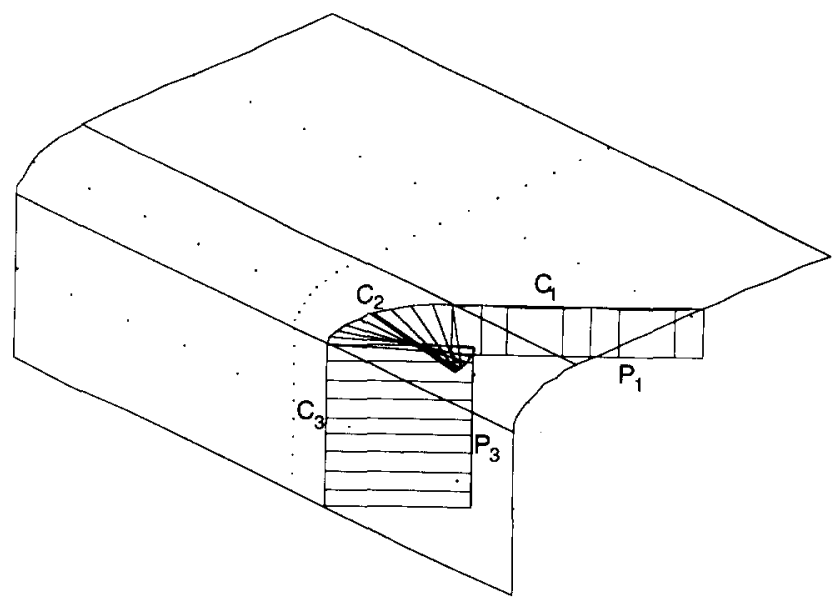

Figure 14 Degenerate case in which all normals intersect 
- Step 2: Remove $\kappa$, and trim the corresponding portion of the offset curve (i.e. the self-intersecting loop).

- Step 3: Recreate the new set of nonintersecting line segments $\boldsymbol{\kappa}^{\prime}$.

- Step 4: Modify the design curve $\mathbf{C}$ by interpolating the values of $R(u)$ that define the trimmed point

Let the composite design curve be denoted $\mathbf{C}=\left\{\mathbf{C}_{1}, \mathbf{C}_{2}\right.$, $\left.\mathbf{C}_{3}, \ldots, \mathbf{C}_{n}\right\}$, and the corresponding offset curve be denoted $\mathbf{P}=\left\{\mathbf{P}_{1}, \mathbf{P}_{2}, \mathbf{P}_{3}, \ldots, \mathbf{P}_{n}\right\}$. Note that each component curve $\mathbf{C}_{\boldsymbol{i}}$ corresponds to the intersection of the cutting plane $T$ with the design surface patch $S_{i}(r, s)$. Below, we describe briefly the details of the modification procedure for a single curve component $\boldsymbol{C}_{i}$.

In Step 1, it is necessary to identify $\boldsymbol{\kappa}$, the set of curve normals that intersect (see Figure 15a). For the design curve $\mathbf{C}_{i}$ and the corresponding offset curve $\mathbf{P}_{i}$, we check whether the line segment $\mathbf{C}_{i}(u) \mathbf{P}_{i}(u)$ intersects with the line segment $\mathbf{C}_{i}(u-1) \mathbf{P}_{i}(u-1)$. Further, it is also necessary to check for the intersection of curve normals at the endpoints of the adjacent curves $\mathbf{C}_{i}$ and $\mathbf{C}_{i+1}$. We adopt the simple strategy outlined in Reference 2.

In Step 2, we remove all the points $\mathbf{P}\left(u^{*}\right)$ that correspond to the intersecting curve normals in Step 1 (see Figure 15b). By removing such points, we also remove the offset curve segment $\mathbf{P}^{*}$ between $\mathbf{P}\left(u_{k}\right)$ and $\mathbf{P}\left(u_{k+n}\right)$. Therefore, after the loop removal, points $\mathbf{P}\left(u_{k}\right)$ and $\mathbf{P}\left(u_{k+n}\right)$ are coincident. Note that we also consider all the removed points to be coincident at $\mathbf{P}\left(u_{k}\right)$. In Step 3, we replace the intersecting curve normals by a new set of line segments $\boldsymbol{\kappa}^{\prime}$ such that each coincident point at $\mathbf{P}\left(u_{k}\right)$ corresponds to a distinct line segment (see Figure $15 \mathrm{c}$ ).

The final step is to modify $\mathrm{C}^{*}$, the portion of the design curve that corresponds to the offset-curve portion $\mathbf{P}^{*}$ that was trimmed. Recall that this is a situation in which the bend radius of the sheet metal is inappropriate for the selected thickness of material. Therefore, the procedure automatically modifies the design-curve portion $\mathrm{C}^{*}$ as a guide to the designer. On the new line

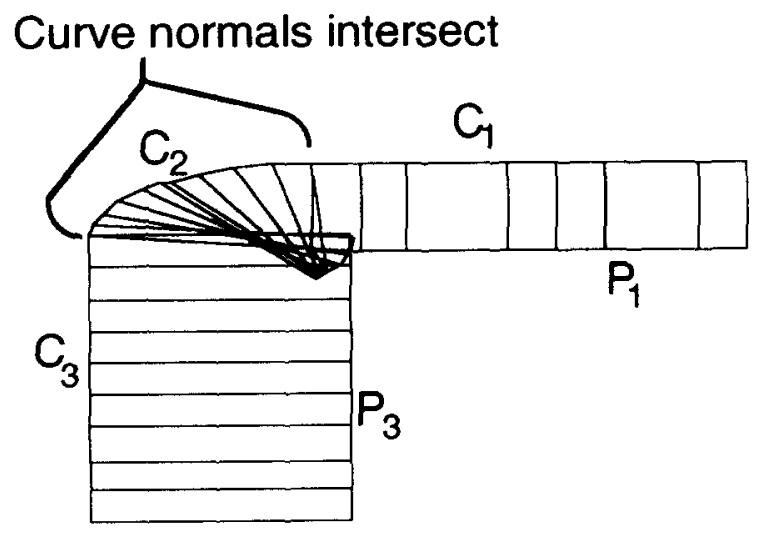

(a)

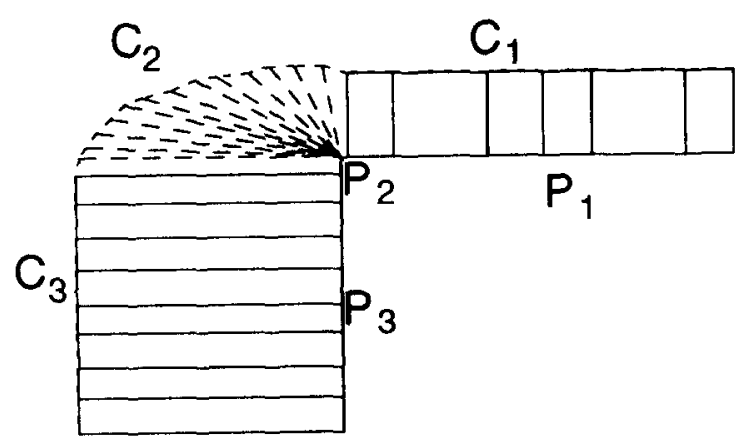

(c)

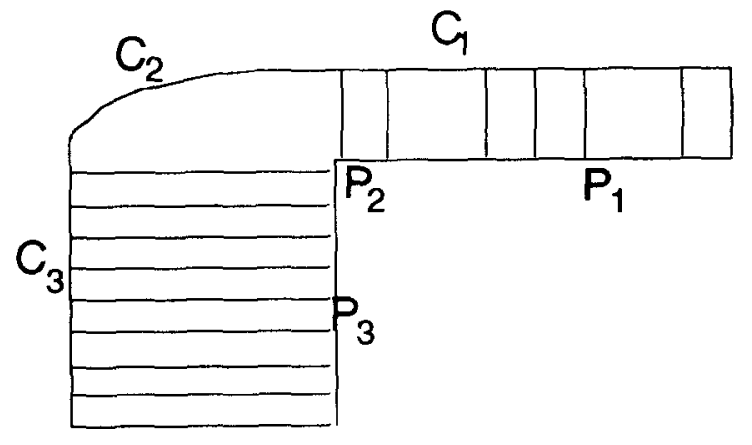

(b)

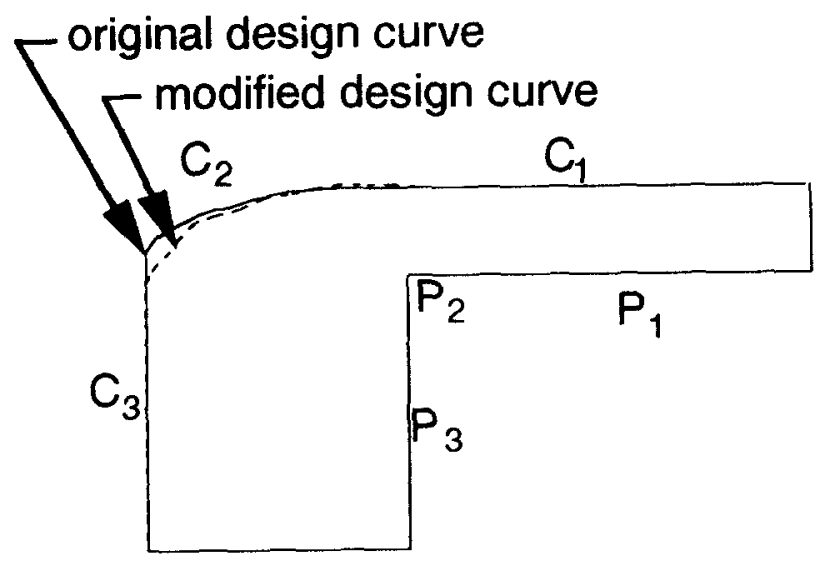

(d)

Figure 15 Stepwise procedure for checking degeneracy 
segments $\boldsymbol{\kappa}^{\prime}$, we select points at distances that interpolate $\lambda_{k}$ and $\lambda_{k+n}$, the distances of the design-curve points endpoints $\mathbf{C}\left(u_{k}\right)$ and $\mathbf{C}\left(u_{k+n}\right)$ from the offset point $\mathbf{P}\left(u_{k}\right)$ (see Figure 15d). In our implementation, we used a linear interpolation:

$$
\boldsymbol{\kappa}^{\prime}\left(u_{i}\right)=(v) * \lambda_{1}+(1-v) * \lambda_{2} \quad i=1,2, \ldots, n
$$

where $v=1 / n$, and $\boldsymbol{\kappa}^{\prime}\left(u_{i}\right)$ is the new line segment $\mathbf{P}\left(u_{i}\right) \mathbf{C}\left(u_{i}\right)$.

\section{EXAMPLES}

The simple L-shaped sheet-metal designs shown in Figures 13 and 14 were implemented using the above modification procedure, and the results are shown in Figures 16 and 17, respectively. Note that, in Figure 17, the curve normals no longer intersect, and $\mathbf{C}_{2}$ is the modified design curve that satisfies the constant-thickness constraint.

Next, we consider a more typical sheet-metal design, as shown in Figure 18. A planar section has been cut through the design surfaces. Using the iterative method outlined above, an offset curve on the outside surface of

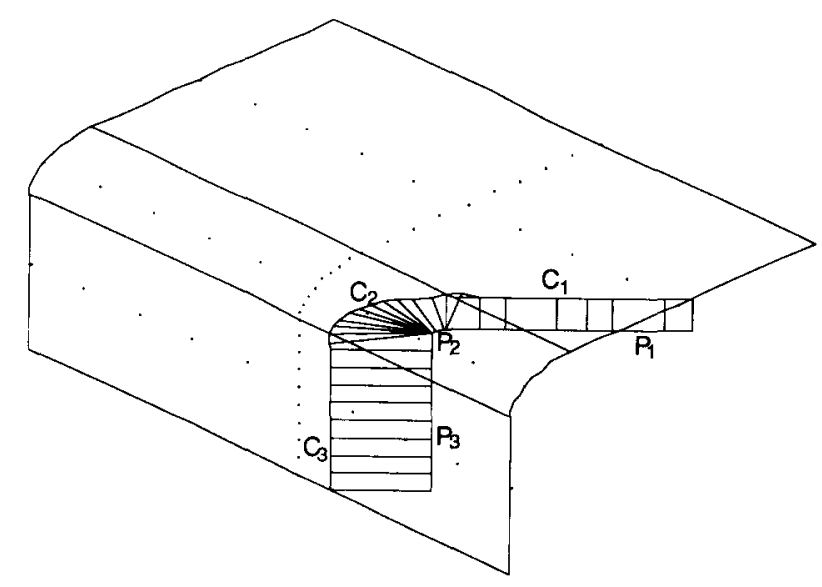

Figure 16 Modification of design shown in Figure 13

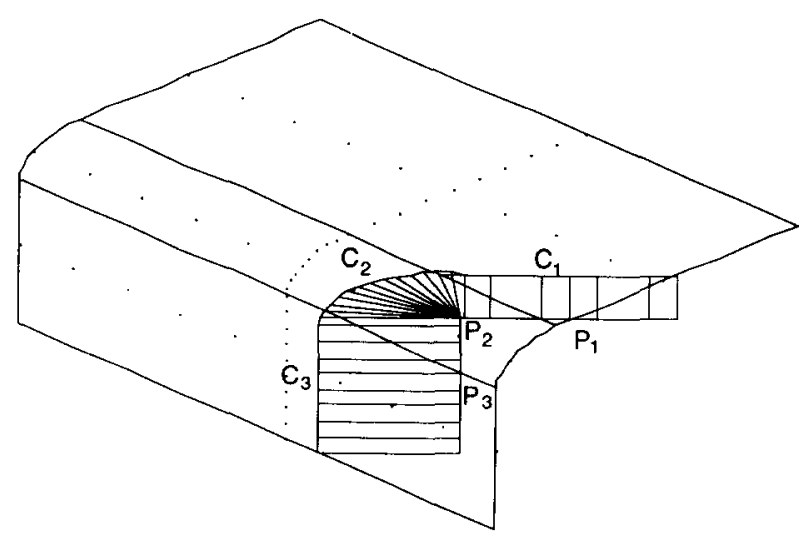

Figure 17 Modification of design shown in Figure 14

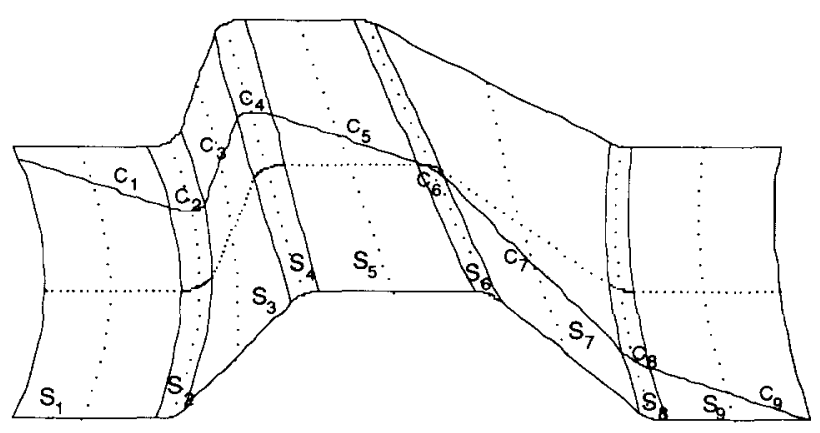

Figure 18 Sheet-metal design

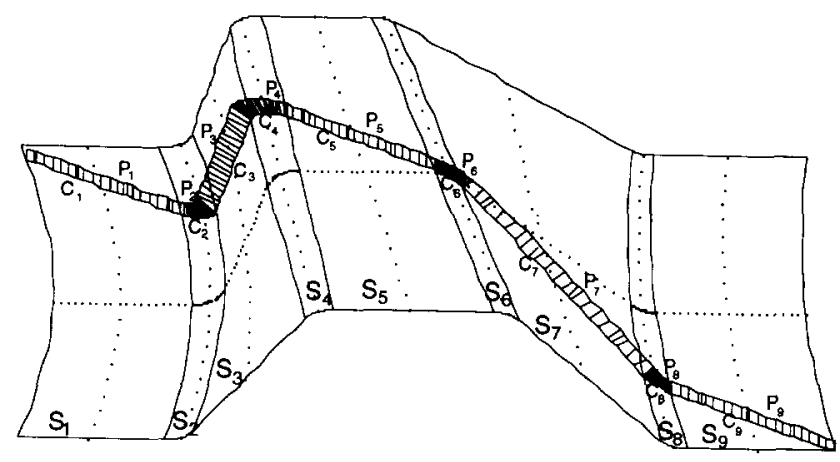

Figure 19 Offset curve on outside surface of metal

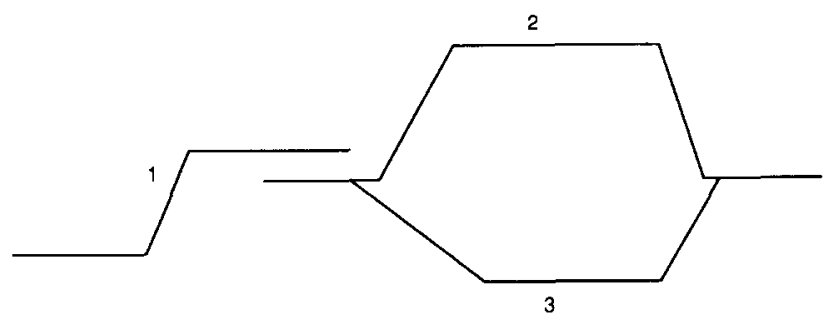

Figure 20 Section of sheet-metal assembly containing three parts

the metal was generated, and this is shown in Figure 19 for a sheet-metal thickness of $2 \mathrm{~mm}$. Line segments $R_{i}(u)$ connecting the design-curve points $\mathbf{C}_{i}(u)$ to $\mathbf{P}_{i}(u)$ are also shown for clarity.

\section{CLEARANCE AND INTERFERENCE CHECKS IN SHEET-METAL DESIGN}

Another practical application for the offset-curve method presented here is that of checking for clearance and interference in sheet-metal assemblies. In sheet-metal products, particularly in automotive applications, many parts are welded together to form an assembly. Since only one side of the sheet metal is designed, it is often difficult to make clearance and interference checks between the different sheet-metal parts within an assembly. Figure 20 shows the planar section of a typical sheet-metal assembly containing three parts 1,2 and 3 . The planar section shows the design side of the sheet 


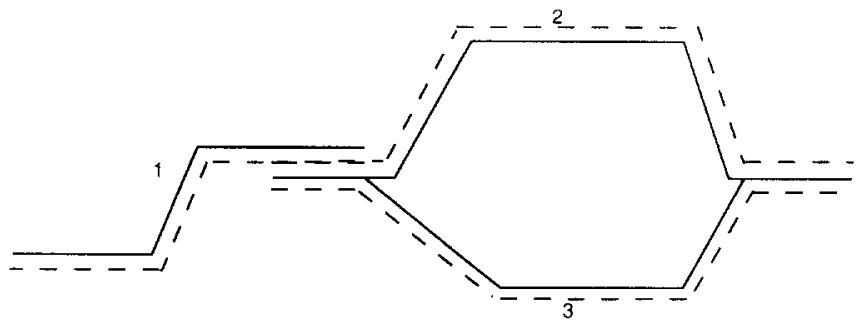

Figure 21 Sheet-metal assembly section with outside of metal showing

metal only. To determine whether any of the parts interfere, or have clearance, it is necessary to cut planar sections through the outside of the metal. This was done using the offset-curve method presented in this paper.

Figure 21 shows the same section with the outside of the metal shown by broken lines. Note that the outside of the metal of part 1 mates with the outside of the metal of part 2, and the inside of the metal of part 2 mates with the inside of the metal of part 3. To check for the interference or clearance for parts 2 and 3 , it is only necessary to look at the design sections. However, for a similar check involving parts 1 and 2 , it is necessary to look at the outside of the metal sections which can be efficiently generated using the offset-curve method.

This interference-checking procedure can be automated by creating an algorithm to cut planar sections at specified intervals, say every $10 \mathrm{~mm}$, for the design surface and the offset surface (outside of the metal). Profiles of the mating part can then be checked against each other for clearance and interference.

\section{CONCLUSIONS}

A simple but practical method of generating offset curves for sheet-metal design has been presented. The method entails iteratively finding the length of the surface normal on the design surface which corresponds to the chosen metal thickness. The method does not involve offsetting surfaces, and the designer can choose the level of accuracy. To handle degenerate cases, the method was modified to include the detection and removal of self-intersecting loops. Some degeneracies require a modification to the design curve which leads to modification of the design surface. Therefore, the method gives a designer the option of modifying the design curve, modifying the metal thickness, or rejecting the calculated offset.

Our method determines the closest planar section that satisfies the offset criteria, and, therefore, it can be used in a variety of sheet-metal design analysis tools. It can also be used to calculate automatically clearance and interference conditions between mating sheet-metal parts. The application of this method might also be possible in layered manufacturing (e.g. stereolithography), where planar sections of the object are required. The planar sections can be cut one at a time, without generating the entire object. This is advantageous, in view of the difficulties associated with creating a solid model from a surfaced sheet-metal part. Finally, this method may be extended to calculate geometrical properties such as the volume and mass of sheet-metal parts.

\section{ACKNOWLEDGEMENT}

Debasish Dutta was supported in part by US National Science Foundation grant DDM-90-10411, and by AFOSR grant 49620-92-J-0095 DEF.

\section{REFERENCES}

1 Hoffmann, C M Geometric and Solid Modeling: An Introduction Morgan Kaufmann, USA (1989)

2 Tiller, $W$ and Hanson, E G 'Offsets of two-dimensional profiles' IEEE Comput. Graph. \& Applic. (Sep 1984) pp 36-46

3 Hoschek, J 'Spline approximation of offset curves' Comput. Aided Geom. Des. Vol 5 (1988) pp 33-40

4 Farouki, R T 'Exact offset procedures for simple solids' Comput. Aided Geom. Des. Vol 2 (1985) pp 257-279

5 Farouki, R T 'The approximation of nondegenerate offset surfaces' Comput. Aided Geom. Des. Vol 3 (1986) pp 15-43

6 Chasen, S H Geometric Principles and Procedures for Computer Graphics Applications Prentice-Hall, USA (1978)

7 Fax, I D and Pratt, M J Computational Geometry for Design and Manufacture Halsted Press, John Wiley, USA (1987)

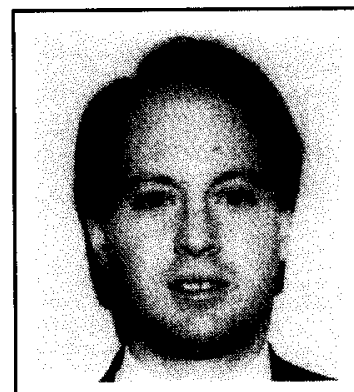

Ken Wentland received a $B S C$ in mechanical engineering at Michigan Technological University, USA, in 1989 In 1989/90, he worked at Chrysler Corporation as an automobile-body design and release engineer. He received an $M S \mathrm{Sc}$ in mechanical engineering at the University of Michigan, USA, in 1992. His research interests are in automobilebody design and manufacturing. While studying at the University of Michigan, he was involved in research relating to variation-reduction methods for automobile sheet-metal assembly.

Debasish Dutta is an assistant professor. He received a PhD from Purdue University, USA, in 1989. His areas of interest include geometric modelling and geometric algorithms for mechanical design and manufacturing. 